\title{
Automatic emotional information processing and the cortisol response to acute psychosocial stress
}

\author{
Mark A. Ellenbogen \\ Concordia University, Montreal, Quebec, Canada \\ Robyn J. CARSON \\ University of Ottawa, Ottawa, Ontario, Canada \\ AND \\ Rana Pishva \\ Queen's University, Kingston, Ontario, Canada
}

\begin{abstract}
Attentional shifting may represent a means of regulating the stress response. Previously, automatic processing of emotional information was predictive of subsequent cortisol levels during a repeated loss stressor (Ellenbogen, Schwartzman, Stewart, \& Walker, 2006). The stress induction did not, however, elicit a substantive cortisol increase. Thus, we sought to replicate this finding using the Trier Social Stress Test (TSST), a validated psychosocial stress induction. Seventy-nine students performed a modified spatial cuing task with supraliminal and masked pictorial stimuli during the TSST $(n=36)$ and a control condition $(n=43)$. The TSST elicited a greater cortisol response than did the control condition $[F(1,76)=4.6, p<.05]$. Attentional shifting during trials with masked angry faces predicted cortisol change during the TSST ( $\beta=.76 ; t=2.1, p<.05)$, but not during the control condition. These data suggest that early automatic emotional information processing is important in the regulation of the cortisol stress response, although the direction of effect is not known.
\end{abstract}

The efficiency and flexibility of early attentional processing is becoming increasingly important in understanding the pursuit of goals (Bargh, 2006) and mental disorders, such as major depression and anxiety disorders (Leppänen, 2006; Mogg \& Bradley, 1998). Emotional biases in information processing and deficient inhibitory filtering may represent key deficits that provide the foundation for disordered thinking, cognitive distortions, memory biases, and high emotionality. These relationships are particularly salient with respect to emotional disorders such as anxiety and depression, where it is now established that there are robust attentional biases specific to these disorders (Ellenbogen \& Schwartzman, 2009; Fales et al., 2008; Gotlib, Krasnoperova, Yue, \& Joormann, 2004; Joormann, Siemer, \& Gotlib, 2007; Mogg \& Bradley, 2005; Sposari \& Rapee, 2007). With some notable exceptions (Calvo \& Avero, 2005; Leyman, De Raedt, Schacht, \& Koster, 2007), attentional biases in anxious and depressed participants are largely attributable to difficulties in disengaging attention from threatening and sad stimuli, respectively, rather than to the initial orienting to these stimuli (Amir, Elias, Klumpp, \& Przeworski, 2003; Ellenbogen \& Schwartzman, 2009; Fox, Russo, Bowles, \& Dutton, 2001; Koster, Crombez, Verschuere, Van
Damme, \& Wiersema, 2006; Koster, De Raedt, Goeleven, Franck, \& Crombez, 2005; Salemink, van den Hout, \& Kindt, 2007). Although this body of literature is of great importance, much of this work has been descriptive, and attempts to understand the functional consequences of attentional and memory biases in emotional disorders have been sparse. The general view is that attentional biases represent precursors to cognitive distortions (i.e., biased appraisals and attributions) and memory retrieval biases that underlie anxiety and depression (Ingram, Miranda, \& Segal, 2006; Mogg \& Bradley, 1998). However, attentional processing may underlie more general mechanisms of self-regulation and regulatory deficits that occur in psychopathology (Posner \& Rothbart, 1998).

Current models of psychopathology identify deficits of emotion regulation as central to our understanding of different mental disorders (Campbell-Sills \& Barlow, 2007; Kring \& Werner, 2004; McLaughlin, Mennin, \& Farach, 2007). In an influential theory of emotion regulation (Gross, 1998, 2002), the process of modulating emotional reactivity is hypothesized to occur through different techniques that can be categorized as "antecedent-" and "response-focused," depending on whether the strategy is applied prior to or during the generation of an emotion, 
or following the emotion. Among the antecedent-focused techniques, attentional allocation was put forth as an important means of emotion regulation. Indeed, studies have demonstrated a robust link between measures of selective attention and the emotional response to laboratory-induced and naturalistic stress (Beevers \& Carver, 2003; Compton, 2000; Ellenbogen, Schwartzman, Stewart, \& Walker, 2006; Mathews \& MacLeod, 2002). Less is known, however, about the biological response to stress.

The hypothalamic-pituitary-adrenal (HPA) axis plays a pivotal role in the organism's adaptation to various forms of biopsychosocial challenge (McEwen, 2004). Among its wide-ranging effects on the brain and behavior, the release of glucocorticoids during stress can influence different facets of information processing (Lupien \& McEwen, 1997), including selective attention (Fehm-Wolfsdorf et al., 1993; Mölle, Albrecht, Marshall, Fehm, \& Born, 1997; Skosnik, Chatterton, Swisher, \& Park, 2000), threat-related information processing (Putman, Hermans, Koppeschaar, van Schijndel, \& van Honk, 2007), and the consolidation of memory of emotionally arousing events (Buchanan \& Lovallo, 2001; Putman, van Honk, Kessels, Mulder, \& Koppeschaar, 2004). Less is known, however, about how information processing influences the activation of the HPA response during a stressful challenge and the regulation of cortisol levels. Importantly, this issue may be fundamental in understanding depression, in that the HPA axis is dysregulated at a number of levels in this disorder (Holsboer, 1995; Young, 2004).

Some studies have found that complex and elaborative cognitive processes, such as cognitive appraisal and perceived coping ability, are important determinants of the HPA response to a psychosocial challenge (Breier, 1989; Gaab, Rohleder, Nater, \& Ehlert, 2005; Lazarus \& Folkman, 1984; van Eck \& Nicolson, 1994; Wirtz et al., 2007). In contrast, few studies have investigated the influence of earlier stages of information processing on the HPA response to stress. In a study using a pictorial version of the emotional Stroop task, selective attention to masked pictures of angry faces was positively associated with the magnitude of cortisol change from pre- to posttask performance (van Honk et al., 2000). Unfortunately, the study did not examine the stress response per se, since participants were not exposed to a stressful challenge. Another study reported that high cortisol responders to a socialevaluative stressor displayed greater attentional vigilance toward masked angry faces than did low cortisol responders (Roelofs, Bakvis, Hermans, van Pelt, \& van Honk, 2007). However, cortisol levels were not correlated with attentional performance, suggesting that the effect could be due to another stress-related factor. Moreover, the results are based on a small sample size $(n=20)$, who were separated into groups by median split. Recently, cortisol reactivity to a social stressor was positively associated with an attentional bias toward negative rejection-related words in a sample of 20 students (Dandeneau, Baldwin, Baccus, Sakellaropoulo, \& Pruessner, 2007).

We have reported, using a modified spatial cuing task (Stormark, Nordby, \& Hugdahl, 1995), that the rapid disengagement of attention from negative stimuli during stress was associated with a lesser mood and salivary cortisol response to stress (Ellenbogen, Schwartzman, Stewart, \& Walker, 2002, 2006). Moreover, the relationship between disengagement and indices of the stress response differed by the stage of information processing (Ellenbogen, Schwartzman, et al., 2006). Among the different facets of information processing, one well-known distinction in the cognitive literature is between automatic and controlled modes of processing (Bargh, 1989; Logan, 1992; Shiffrin \& Schneider, 1977). The former is characterized by a fast and almost limitless capacity in processing information, requiring minimal effort, intention, or subjective awareness. Controlled processing is subject to limitations in capacity and requires effort, intention, and subjective awareness. Although the categorization of information processing into automatic and controlled is somewhat arbitrary, it represents a useful framework for exploring different stages of information processing. In our previous study (Ellenbogen, Schwartzman, et al., 2006), slow disengagement from supraliminal dysphoric pictures was associated with a greater negative mood response, but had no impact on cortisol levels. In contrast, slow disengagement from masked pictures depicting threat (presented with limited conscious awareness) was predictive of greater cortisol change, but had no effect on self-rated mood. Thus, effortful or controlled processing of dysphoric pictures was associated with mood regulation, whereas the automatic processing of threatening pictures was associated with the regulation of cortisol levels. Automatic processing of evolutionarily significant information, occurring prior to conscious awareness, may be sufficient to activate physiological indices of the fear response through a direct neural circuit between the thalamus and the lateral nucleus of the amygdala (LeDoux, 2002; Öhman, Carlsson, Lundqvist, \& Ingvar, 2007). For this reason, the early and rudimentary analysis of threat-related stimuli may be closely coupled with subcortical defensive systems involving the amygdala, such as the HPA axis.

In the present study, we sought to replicate our previous finding that the speed of disengagement from masked pictures depicting threat was predictive of subsequent cortisol levels during a repeated loss stressor (Ellenbogen, Schwartzman, et al., 2006). The stress induction used in the previous study involved participants losing 9 out of 12 computer games and earning no money against a confederate posing as another participant. The major limitation of the study was that, despite robust mood change, cortisol levels were not substantially altered by the stress induction. Moreover, despite the robust relationship between disengagement and cortisol levels overall, there was no evidence that attentional disengagement moderated the effect of the stress induction on cortisol levels per se, because no interaction between condition and disengagement was observed. The results suggested that slow disengagement from stimuli depicting threat was associated with greater cortisol levels in general, perhaps reflecting a stable relationship (i.e., trait) that is independent of stress. Thus, the present study utilized the Trier Social Stress Test (TSST), a well-established psychosocial stress induction known to activate the HPA axis and increase cortisol levels (Dicker- 
son \& Kemeny, 2004; Kirschbaum, Pirke, \& Hellhammer, 1993). In this stress induction, participants performed a public speech and mental arithmetic in front of a panel of "experts" (confederates) and video-recording equipment. During the stress induction, participants performed a modified spatial cuing task (Ellenbogen et al., 2002; Stormark et al., 1995) using sad, angry, and neutral facial expressions presented at different exposure durations (200 and $750 \mathrm{msec}, 17 \mathrm{msec}$ followed by a mask). The brief exposure duration $(17 \mathrm{msec})$ with a mask was meant to restrict conscious awareness of the picture and to assess automatic processing of emotional information. The long exposure duration $(750 \mathrm{msec})$ was meant to measure effortful processing. We chose these presentation times because they were used in our previous study of attentional shifting and cortisol levels during a stressful challenge, where we found specific relationships between automatic and effortful processing and different indices of the stress response (Ellenbogen, Schwartzman, et al., 2006). We also included a 200-msec exposure duration in the present study, which was meant to assess an early stage of information processing, but with full conscious awareness.

Spatial cuing assesses the latency to respond to a neutral target following either a valid or invalid cue (Posner, Snyder, \& Davidson, 1980). Cues are valid when presented in the same hemifield as the target, and they are invalid when presented to the contralateral hemifield of the target. Valid cuing is believed to assess shifts of spatial attention from fixation to the cue and the allocation of attention at that location - also referred to as "attentional engagement." Invalid cuing necessitates participants to first shift to the location of the cue, and then to disengage from that location and move attention to contralateral visual space where the target stimulus appears. This process, referred to as "disengagement," incurs a cost that can be measured as a delay in reaction time (RT) for invalid relative to valid trials. Thus, the valid versus invalid manipulation permits differentiation in RT between the engagement and disengagement components of spatial attention (Luck et al., 1994; Posner, Nissen, \& Ogden, 1978). In the present study, we used attentional costs (also known as the "cue validity index") to assess the efficiency of attentional shifting. Attentional costs are computed by subtracting the RT on valid trials from the RT on invalid trials for neutral and emotional stimuli. In our past research using an index of disengagement (Ellenbogen \& Schwartzman, 2009; Ellenbogen, Schwartzman, et al., 2006), valid trials, which comprise $78 \%$ of all trials, were excluded from the analyses. In computing attentional costs, all trials were utilized, which provided a more comprehensive, stable, and reliable measure of attentional shifting efficiency than did the response to valid (engagement) and invalid (disengagement) trials alone. In general, attentional costs during the spatial cuing task are greatest when participants are both fast to shift toward the emotional cue and slow to disengage from the cue. The examination of attentional costs has uncovered meaningful differences between, for example, depressed and nondepressed samples (Koster et al., 2005; Leyman et al., 2007) and has been purported by some to be a measure superior to indices of disengagement (Mogg, Holmes, Garner, \& Bradley, 2008).
A related goal of the present study was to assess the generalizability of the relationship between automatic processing and HPA functioning. The previous study utilized a community sample of depressed, anxious, and control participants, and pictorial stimuli depicting scenes of sadness (i.e., a crying baby, a sad face, and an injured animal) and threat (i.e., guns and scenes of violence). In the present study, we assessed whether this relationship was present in a nonclinical sample of university students, using pictures of facial expressions of emotion. Two hypotheses were put forth. First, as was outlined previously, we hypothesized that the interaction between attentional costs during trials with masked angry faces and the experimental condition would significantly predict cortisol change during the stress induction. In other words, the greatest changes in cortisol levels were expected to occur in those participants who were exposed to the stressor and who exhibited high attentional costs (i.e., poor attentional flexibility) to masked pictures of angry faces. Second, on the basis of previous studies of attentional shifting (Ellenbogen \& Schwartzman, 2009; Ellenbogen, Schwartzman, et al., 2006; Fox et al., 2001), we predicted that the aforementioned relationship would largely be driven by abnormalities in the process of disengaging attention from an emotional face, rather than by the process of engaging attention at the cued location.

\section{METHOD}

\section{Participants}

Eighty-five participants, 18-34 years of age, were recruited through classroom visits and advertisements that were posted in university newspapers. Using an in-house screening interview, potential participants were interviewed over the telephone. Candidate participants were excluded for reasons of pregnancy/lactation, color blindness, current use of a glucocorticoid medication, and the presence of a current mental disorder or any major medical condition. Six participants (control, 2; TSST, 4) provided too few or no saliva samples (i.e., difficulties spitting, terminated before completing the study, laboratory error) and were dropped from the study. Thus, 79 university students participated in the study; 36 were randomly assigned to the TSST, and 43 to the control condition. Participants included 44 women (control, 24; TSST, 20) and 35 men (control, 19; TSST, 16), with a mean age of 22.8 years \pm 3.6 (control, $22.5 \pm 3.5$; TSST, $23.1 \pm 4.1$ ). Eleven participants reported smoking (control, 6; TSST, 5), 9 reported medication use (control, 7; TSST, 2), and 13 reported oral-contraceptive use (control, 10; TSST, 3$)$. Medications reported ( $n=1$, except when indicated) included ibuprofen, acetaminophen/dextromethorphan/ pseudoephedrine (Tylenol Flu), isotretinoin (Accutane for acne), cyproterone-ethinyl estradiol (Diane-35 for acne), metformin (antidiabetic, $n=2$ ), oxycontin, levothyroxine sodium (Synthroid for thyroid condition), a dieting pill (unknown), and a nonglucocorticoid treatment for dry skin (unknown).

\section{Measures}

Current symptoms of depression and anxiety were assessed using the Beck Depression Inventory-II (Beck, Steer, \& Brown, 1996) and the Penn State Worry Questionnaire (Meyer, Miller, Metzger, \& Borkovec, 1990). Mean depression $(n=78)$ and anxiety $(n=74)$ scores in the total sample $( \pm S D)$ were $10.3 \pm 8.3$ $($ range $=0-36)$ and $47.5 \pm 14.8$ (range $=21-79)$, respectively. No differences in depression and anxiety were observed between participants in the control (depression, $9.9 \pm 7.8$; anxiety, $47.7 \pm$ 14.5) and TSST (depression, $10.7 \pm 9.0$; anxiety, $47.2 \pm 15.5$ ) conditions. 


\section{Modified Spatial Cuing Task}

In this adapted stimulus detection task (Ellenbogen et al., 2002; Stormark et al., 1995), participants are requested to fixate on a centrally placed gray "+" sign on a black background, which is flanked on both sides by a gray rectangle $(3.7 \times 3.2 \mathrm{~cm})$. They are required to respond with a single keypress as fast as possible when the target (a black dot) appears in either of the rectangles. Preceding all target presentations, a cue appears in one of the rectangles. The cue is a picture of a sad, angry, or neutral face that signals the likely location of the target on each trial. There were 380 trials, divided into 15 blocks ( 5 blocks for each picture category) of 24 trials, and 20 additional "catch" trials presented across the blocks, in which the target did not appear following the presentation of the cue. Catch trials were meant to prevent participants from developing an automatic response set due to the fixed cue-target intervals in this experiment, and they were not included in the statistical analyses. On valid or attentional engagement trials (270), the cue and target appeared in the same hemifield. On invalid or disengagement trials (90), the cue and target appeared in opposing hemifields. The stimulus onset asynchrony (the interval between the onset of the cue and onset of the target) for valid and invalid trials was 833 or $283 \mathrm{msec}$, and the interval between trials (from the offset of the target to next cue onset) was 1.85 to $2.5 \mathrm{sec}$. Cues (pictures) were presented for 750 or $200 \mathrm{msec}$, or $17 \mathrm{msec}$ followed by a masking stimulus. For masked trials, the mask was presented for $183 \mathrm{msec}$ immediately at the offset of the cue. At $83 \mathrm{msec}$ following the offset of the cue or mask, targets were presented for $600 \mathrm{msec}$.

Participants performed the task using a chinrest that was $57 \mathrm{~cm}$ away from the monitor. The center of each rectangle was $2.2^{\circ}$ of visual angle from the fixation point. Within each block, the picture cues were of the same emotional valence in order to avoid affective carryover effects, in which the emotional valence of the cue on one trial influences the response on the subsequent trial. The trials within a block varied in terms of the trial type (750 or $200 \mathrm{msec}$ supraliminal, or masked), and stimulus presentations were distributed equally between the right and left visual hemifields. The order of blocks was varied randomly across participants, except that two blocks of the same picture category were never consecutive. Validly and invalidly cued targets (including catch trials) represented $75 \%$ and $25 \%$ of all trials, respectively - a ratio shown to be effective in cuing attention (Posner, 1978). RTs less than $150 \mathrm{msec}$ and more than $850 \mathrm{msec}$ were excluded from the analyses.

Three indices of attentional shifting were computed from RT data on the modified spatial cuing task. First, attentional costs were computed by subtracting the RT for valid trials from the RT for invalid trials for each picture category. Positive scores (i.e., high attentional costs) were attributed to slow RT during invalid cuing trials and/or fast RT on valid trials. Negative scores and scores close to 0 (i.e., low attentional costs) were attributed to slow RT on valid trials and/or rapid RT on invalid trials. In the present study, we presented the relative attentional costs by subtracting the costs during neutral trials from those during trials with angry and sad faces. Second, the RT data were also converted into disengagement scores by subtracting the RT for invalid trials with neutral faces from the RT for invalid trials with sad and angry pictures. Third, engagement scores were computed by subtracting the RT for valid trials with angry or sad faces from the RT for valid trials with neutral faces. In both cases, positive scores indicate increased selective attention for negative stimuli, and negative scores indicate attentional avoidance of negative stimuli. Spatial cuing data were collapsed across hemifield of presentation.

Pictures were selected from the Pictures of Facial Affect database (Ekman \& Friesen, 1976), which contains 110 pictures of facial expressions that have been widely validated and used in cross-cultural studies. The male and female facial pictures were black and white and depicted angry, sad, or neutral facial expressions. In total, the 36 pictures (12 per picture category) with the highest percentage ratings of emotional or neutral affect were selected for the spatial cuing task. Pictures were scaled to the same size as the background rectangles $(3.7 \times 3.2 \mathrm{~cm})$, using a graphic editing software. Masks were made by cutting pictures into small pieces and randomly reassembling them. The backward masking procedure with pictures has been effectively used in other studies of automatic or preattentive processing (Öhman \& Soares, 1994). The modified spatial cuing task was performed on an IBM PC computer, with a 17-in. NEC color monitor. The task was programmed using the STIM Stimulus Presentation System software (Version 7.584) developed by the James Long Company (Caroga Lake, NY).

\section{Awareness Check}

A computerized awareness check was administered in which participants were asked to indicate (or guess) whether the face presented just before the masking stimulus was male or female. Twenty-four (12 of each category) masked pictures were presented for $17 \mathrm{msec}$, followed immediately by a mask for $183 \mathrm{msec}$. Participants then responded with a two-choice keypress.

\section{The TSST}

The TSST consists of a 10-min period to prepare a speech, a 5 -min public speech, and 5 min of mental arithmetic (Kirschbaum et al., 1993). Both tasks were performed in front of audio and video recording equipment and a committee of two expert "judges" (confederates) who were introduced as specialists in nonverbal behavior. Each participant was asked to deliver a speech as if he or she had applied for a position and was invited by that institution (corporation, school, department, etc.) to present his or her case. If participants did not speak for the 5-min period, then confederates posed questions such as "What qualifies you in particular for this position?" or "What makes you a better candidate than your classmates?" The mental arithmetic task consisted of counting aloud backward from 2,083 to 0 by intervals of 13 . Participants were asked by the committee members to start from the beginning after each mistake.

\section{Measurement of Salivary Cortisol}

Saliva was expressed directly into polypropylene 6-ml vials. Samples were frozen at $-20^{\circ} \mathrm{C}$ until they were assayed for cortisol using a sensitive commercial enzyme immunoassay kit from Salimetrics (State College, PA; Schwartz, Granger, Susman, Gunnar, \& Laird, 1998). The sensitivity of the assay was set at $0.012 \mu \mathrm{g} / \mathrm{dl}$. The interand intra-assay coefficient of variation for the assays were $3.9 \%$ and $4.6 \%$ (on a range of $0.01-10 \mu \mathrm{g} / \mathrm{dl}$ dose), respectively. Assays were conducted in the laboratory of C.-D. Walker at the Douglas Hospital Research Centre (Montreal, Canada).

\section{Procedure}

Following a telephone screening, participants refrained from eating and drinking (except water) $1 \mathrm{~h}$ prior to arrival at the laboratory and during the experimental procedures. They arrived at the laboratory at either $12: 15$ or 15:00 h, provided informed consent, and then underwent $30 \mathrm{~min}$ of relaxation. In a dimly lit room, participants reclined in a comfortable chair, listening to relaxing music and/or reading. They were then randomly assigned to the TSST (described previously) or a control session. Participants in the TSST condition completed the public speech and $2.5 \mathrm{~min}$ of the mental arithmetic before beginning a testing phase that included the modified spatial cuing task (approximately $40 \mathrm{~min}$ ), a test of awareness (to test the validity of the masking procedures, approximately $5 \mathrm{~min}$ ), and a visual search task (approximately $15 \mathrm{~min}$, not presented here). The spatial cuing and visual search tasks were presented in random order, but the awareness test was always conducted last. At the end of these procedures, participants then completed the remainder of the TSST protocol, consisting of $2.5 \mathrm{~min}$ of mental arithmetic. In the control condition, participants watched a neutral video presentation (documentary about language) for the length of time during which participants were exposed to the first part of the TSST, and then completed the information-processing tasks. At the end of the information processing tasks, they filled out a bogus documentary rating scale about the video presentation to ensure that the participants had actually watched the video. At the end of the TSST and control 
sessions, participants underwent a 40-min recovery phase, during which they reclined in a comfortable chair, listening to music and/ or reading. Questionnaires were administered, beginning at $30 \mathrm{~min}$ into the recovery phase (only two are reported here).

Mood ratings were recorded on four different occasions throughout the experiment (not reported here). Saliva was collected on 12 different predetermined occasions, but the first two samples were excluded (prerelaxation, postrelaxation) from the study because salivary cortisol levels were very high and declining during this period. These samples tend to be subject to outside influences (i.e., driving, public transport, novelty of the situation). Thus, saliva measures in the present study were as follows: at the end of speech preparation (Sample 1, baseline), at the end of the first mental arithmetic (Sample 2 ), at $+15,+30$, and +45 min into the information processing tasks (Samples 3-5), at the end of the second mental arithmetic (Sample 6), and at $+10,+20,+30$, and +40 min into the recovery period (Samples 7-10). Sampling in the control session occurred at the same time points, except that Samples 1 and 2 occurred after 10 and $17.5 \mathrm{~min}$ from the beginning of the video presentation, and Sample 6 occurred after participants had filled out the bogus documentary rating scale. In total, the procedures lasted approximately $2.5 \mathrm{~h}$. Participants were compensated $\$ 30 \mathrm{CAN}$ for time spent in the laboratory. All of the procedures were approved by the Concordia University Research Ethics Committee.

\section{Data Analysis}

To correct for positive skewness, a log transformation was performed on the cortisol data. Statistical analyses were performed on transformed data, but the figures and tables present the original data for interpretation purposes. Three outlier cortisol values (defined as being over $4 S D$ s above the mean) in 1 participant were detected during the TSST: $0.93 \mu \mathrm{g} / \mathrm{dl}$ (Sample 2), $1.42 \mu \mathrm{g} / \mathrm{dl}$ (Sample 3), and $2.63 \mu \mathrm{g} / \mathrm{dl}$ (Sample 4). Because the values were physiologically possible and were not associated with laboratory error or any evident confound, they were corrected by reducing their values to approximately $4 S D$ s from the mean. The outliers positively skewed the data even after logarithmic and other types of transformations. The corrected outlier values maintained the same rank order in the data as the noncorrected values, and improved the sample distribution. To assess whether cortisol levels, in micrograms per deciliter $(\mu \mathrm{g} /$ dl), differed by experimental condition, the data were subject to a mixed-design ANCOVA, controlling for the time of the first sample (in minutes after midnight). Cortisol output was also examined using the area under the curve "with respect to ground" (AUCg), measuring total hormonal output, and the area under the curve "with respect to increase" (AUCi), a reactivity measure sensitive to changes over time (Pruessner, Kirschbaum, Meinlschmid, \& Hellhammer, 2003). Samples 9 and 10 were excluded from the calculation of AUC, since they showed almost no change from Sample 8 (see Figure 1). Thus, AUC measures were based on Samples 1-8.

The hypothesis that attentional costs and disengagement would moderate the cortisol response to stress was examined using hierarchical multiple regressions. The regressions examined the relative contributions of the time of the first saliva sample, experimental condition, attentional shifting measures (costs, disengagement) for both sad and angry faces, and the interactions between condition and attentional shifting. Significant interactions were followed up with tests of simple slope, which assess whether the slope depicting the relationship between stressor manipulation and cortisol change is significantly different from 0 in participants who are high and low on attentional costs. Simple slope analyses were conducted following the procedures described by Aiken and West (1991). Because interaction terms were included in the regression models, attentional costs were centered (transformed to residual scores) to reduce multicollinearity among predictors (Aiken \& West, 1991). Gender was initially analyzed as an independent variable for the ANCOVAs, but was found to have no main effects or important interactions, and was subsequently dropped from all further analyses. Estimates of

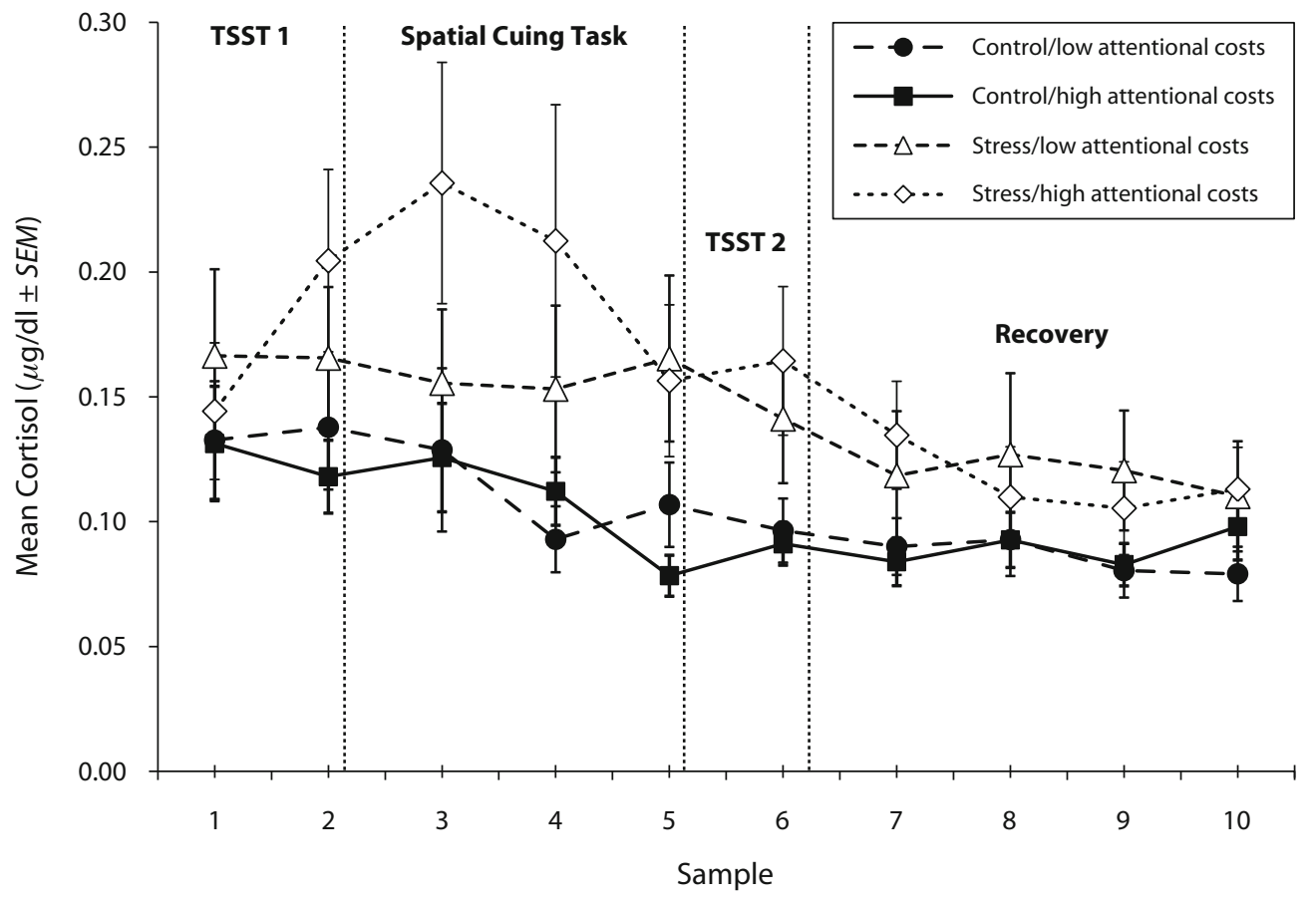

Figure 1. Mean cortisol levels during each phase of the experiment for participants undergoing either the Trier Social Stress Test (TSST) or a neutral control condition. Participants are grouped as those above (high attentional costs, $n=40$ ) and below (low attentional costs, $n=39$ ) the 50th percentile of the distribution of attentional cost scores for masked angry faces. Participants in the stress condition with high attentional costs for masked angry faces exhibited a greater cortisol response than did all other groups. 
effect size are reported for all ANOVAs $\left(\eta_{\mathrm{p}}^{2}\right)$ and regressions $\left(R^{2}\right.$ and adjusted $R^{2}$ ).

\section{RESULTS}

\section{Effectiveness of the Stress Induction}

A two-way (experimental condition $\times$ sample) mixeddesign ANCOVA (controlling for the time of the first saliva sample) on mean cortisol levels revealed a significant condition $\times$ sample interaction $[F(9,684)=1.95, p<.05$; $\left.\eta_{\mathrm{p}}^{2}=.03\right]$ and a trend for significance for the main effect of condition $\left[F(1,76)=3.9, p=.051 ; \eta_{\mathrm{p}}^{2}=.05\right]$. These data are presented in Figure 1. As was expected, the covariate, the time of the first saliva sample, was a robust predictor of cortisol levels $\left[F(1,76)=13, p<.005 ; \eta_{\mathrm{p}}^{2}=.15\right]$. To follow up the condition $\times$ sample interaction, trend analyses (polynomial within-subjects contrasts) and planned comparisons were performed on the samples following the first part of the TSST (Samples 1-5). These analyses revealed a significant quadratic effect $\left[F(1,35)=4.8, p<.05 ; \eta_{\mathrm{p}}^{2}=\right.$ .12] during the stress condition, but not during the control condition (see Figure 1). The quadratic effect indicates that cortisol levels displayed an inverted U-shaped pattern during the first part of the TSST. Moreover, cortisol levels at Samples $2\left[F(1,35)=5.7, p<.05 ; \eta_{\mathrm{p}}^{2}=.14\right]$ and $3\left[F(1,35)=5.0, p<.05 ; \eta_{\mathrm{p}}^{2}=.13\right]$ were significantly higher than the first saliva sample collected during the stress condition, but not during the control condition. Analyses were conducted on cortisol levels in response to the second phase of the TSST (Samples 5, 6, and 7), and they revealed no stress-related increases in cortisol.

Cortisol levels were also examined using AUCg (total output) and AUCi (change over time). An ANCOVA (controlling for the time of sampling) revealed a main effect of condition for both $\mathrm{AUCg}\left[F(1,76)=5.8, p<.05 ; \eta_{\mathrm{p}}^{2}=\right.$ $.07]$ and AUCi $\left[F(1,76)=4.6, p<.05 ; \eta_{\mathrm{p}}^{2}=.06\right]$. Cortisol levels were greater during the TSST than during the control condition (see Table 1). In sum, these analyses indicate that cortisol levels significantly increased following the first part of the TSST, but not following the second part, relative to those in a neutral control session.

\section{Predicting Cortisol Change During the Stress Induction}

Attentional costs: Masked stimuli. For these analyses, relative attentional costs for pictures of angry and sad faces were used (see the Spatial Cuing section for a description). These data, as well as cortisol AUC values, are presented in Table 1. Hierarchical multiple regressions were conducted to assess whether attentional costs for masked pictures depicting threat or sadness moderated the relationship between the stressor manipulation and the magnitude of cortisol change. For AUCg, both the experimental condition and the timing of the first sample were strong predictors of cortisol, but neither attentional costs nor their interaction with condition were significant predictors in the equation (data not shown). Therefore, all subsequent analyses utilized AUCi. Because the timing of the first sample was unrelated to cortisol AUCi $(r=.01$, n.s. $)$, it was dropped from all subsequent analy- ses. Independent variables were entered in the following steps: (1) experimental condition (coded 1 and 2 for the neutral and stress conditions, respectively), (2) attentional costs for masked pictures depicting sadness and threat, and (3) the condition $\times$ attentional costs interaction terms for pictures depicting sadness and threat.

The regression equation predicting cortisol AUCi was significant $(R=.47)[F(5,73)=4.1, p<.005]$, accounting for $22 \%$ (adjusted $R^{2}=17 \%$ ) of the variance (see Table 2). Experimental condition and attentional costs for masked pictures depicting threat and sadness were significant predictors of cortisol $\mathrm{AUCi}$, accounting for $17 \%$ (adjusted $R^{2}=14 \%$ ) of the variance. More importantly, in Step 3, the condition $\times$ attentional costs interaction term for pictures depicting threat, but not sadness, was a significant predictor of the magnitude of cortisol change during the protocol, accounting for an additional 5\% (adjusted $R^{2}=3 \%$ ) of the variance. Although Step 3 fell short of conventional statistical significance (Table 2), repeating the regression after removing the interaction term for pictures depicting sadness, which added nothing to the equation, yielded a statistically significant increment in $R^{2}$ at Step $3\left[F_{\text {inc }}(1,74)=4.1, p<.05\right]$. These data are depicted in Figures 1 (mean cortisol levels) and 2 (AUCi) for attentional costs on trials with angry faces, and in Figure 3 for attentional costs on trials with sad faces. To simplify the presentation of these data in Figures 1-3, a grouping variable depicting "high" and "low" attentional costs was created using a median split of relative attentional costs.

To follow up the significant condition $\times$ attentional costs interaction for angry faces, simple slope analyses were performed (Aiken \& West, 1991). They revealed that high attentional costs ( $1 S D$ above the mean) were associated with increased cortisol change during the TSST relative to the control condition $(t=2.58, p<.05)$ (see Figure 2). In contrast, the slope for low attentional costs ( $1 S D$ below the mean) across conditions did not differ from 0 . In addition, we conducted regressions examining the influence of attentional costs on cortisol AUCi within each of the experimental groups. As was expected, attentional costs for masked pictures depicting anger predicted cortisol change during the TSST $[\beta=.47 ; t=2.5, p<.05]$, but not during the control condition $[\beta=.13 ; t=0.8$, n.s. $]$.

A secondary regression analysis $(n=78)$, as was previously described, was conducted on cortisol AUCi to rule out potential confounds, including smoking (coded as "yes" or "no"), oral contraceptive use, any medication use, and Beck Depression Inventory scores. Except for depression $[\beta=.26 ; t=2.1, p<.05]$, none of the confounds (entered in the first step) were predictive of cortisol AUCi $\left[F_{\text {inc }}(4,73)=1.3\right.$, n.s. $]\left(R^{2}=.07\right.$; adjusted $\left.R^{2}=.02\right)$. More importantly, the condition $\times$ attentional costs interaction term for pictures depicting threat $[\beta=$ .77; $t=2.1, p<.05]$, but not sadness, was predictive of cortisol AUCi, even with the potential confounds in the equation. The addition of the interaction terms at Step 3 yielded a marginally statistically significant increment in $R^{2}\left[F_{\text {inc }}(2,68)=2.4, p=.096\right]\left(R^{2}=.05\right.$; adjusted $R^{2}=$ $.03)$. In sum, two important relationships emerged from these analyses. Consistent with our second hypothesis, 
Table 1

Mean Area Under the Curve (AUC) Cortisol Levels (Arbitrary Units) and Indices of Attentional Shifting (in Milliseconds) by Experimental Condition

\begin{tabular}{lrrrrr}
\hline & \multicolumn{2}{c}{ Stress } & & \multicolumn{2}{c}{ Control } \\
\cline { 2 - 3 } \cline { 6 - 7 } & $M$ & $S D$ & & $M$ & $S D$ \\
\hline Cortisol & & & & \\
AUC with respect to ground & 14.3 & 11.2 & & 9.2 & 6.0 \\
AUC with respect to increase & 1.2 & 8.0 & & -2.1 & 4.9 \\
Indices of Attentional Shifting & & & & & \\
Costs: Masked angry minus neutral & 17 & 37 & & 14 & 48 \\
Costs: Masked sad minus neutral & 22 & 50 & & 15 & 47 \\
Costs: 200 msec angry minus neutral & 0 & 32 & & 1 & 58 \\
Costs: 200 msec sad minus neutral & 4 & 44 & & 11 & 56 \\
Costs: 750 msec angry minus neutral & 8 & 40 & & -3 & 42 \\
Costs: 750 msec sad minus neutral & -5 & 43 & & -5 & 38 \\
Disengagement: Masked angry minus neutral & 18 & 32 & & 18 & 40 \\
Disengagement: Masked sad minus neutral & 20 & 40 & & 20 & 43 \\
Engagement: Masked neutral minus angry & -1 & 17 & & -5 & 21 \\
Engagement: Masked neutral minus sad & 3 & 25 & & -5 & 25 \\
\hline
\end{tabular}

Note-Attentional costs were computed by subtracting reaction time (RT) for valid trials from the RT for invalid trials for each type of picture.

high attentional costs for masked pictures depicting anger were predictive of elevated cortisol reactivity, but only during the TSST. The relationship persisted even after controlling for potential confounding factors. Unexpectedly, low attentional costs for masked pictures depicting sadness were associated with greater cortisol reactivity during the experimental procedures in general.

Attentional costs: 750- and 200-msec exposure durations. Regressions predicting cortisol AUC were repeated using attentional costs for pictures presented at 200- and 750-msec exposure durations. With the exception of experimental condition (data not shown), no significant findings emerged.

Attentional engagement and disengagement. To follow up the findings for attentional costs with masked trials, similar regression analyses of cortisol AUCi were conducted using valid (engagement) and invalid (disengagement) trials as predictors. The regressions were the same as those described previously, except that disengagement and engagement scores (see the Spatial Cuing section for descriptions) replaced attentional costs as predictors. We do not report on the effect of experimental condition in these analyses, since they were the same as those reported previously.

For invalid trials, disengagement scores for masked trials with sad faces were predictive of cortisol AUCi $(\beta=$ $-.280 ; t=-2.7, p<.05$ ), but no significant findings for masked angry faces were observed. For valid trials, attentional engagement scores on trials with masked sad faces $(\beta=-.251 ; t=-2.2, p<.05)\left(\right.$ Step $2 R^{2}=.06$; adjusted $\left.R^{2}=.04\right)$ and the condition $\times$ engagement interaction on trials with masked angry faces $(\beta=.792$; $t=2.4, p<.05)\left(\right.$ Step $3 R^{2}=.07$; adjusted $\left.R^{2}=.05\right)$ were both significant predictors of cortisol AUCi. Simple slope analysis revealed that rapid attentional shifting toward masked angry faces ( $1 S D$ above the mean) was

Table 2

A Hierarchical Multiple Regression $(n=79)$ Predicting Cortisol Area Under the Curve With Respect to Increase During the Laboratory Protocol From Experimental Condition, Attentional Costs, and Their Interaction

\begin{tabular}{lcccccccc}
\hline \multicolumn{1}{c}{ Predictors } & $\mathrm{B}$ & $S E \mathrm{~B}$ & $\beta$ & $t$ & $R$ & $R^{2}$ & Adj. $R^{2}$ & $F_{\text {inc }}$ \\
\hline Step 1: Condition & .014 & .006 & .239 & $2.2^{*}$ & & & & \\
$\quad$ Total step & & & & & .24 & .06 & .05 & $4.7^{*}$ \\
Step 2: Condition & .015 & .006 & .260 & $2.5^{*}$ & & & & \\
$\quad$ Attentional costs: Angry & .177 & .084 & .259 & $2.5^{*}$ & & & & \\
$\quad$ Attentional costs: Sad & -.113 & .073 & -.393 & $-3.2^{* *}$ & & & & \\
$\quad$ Total step & & & & & .41 & .17 & .14 & $5.2^{* *}$ \\
Step 3: Condition & .015 & .006 & .256 & $2.5^{*}$ & & & & \\
$\quad$ Attentional costs: Angry & -.303 & .249 & -.444 & -1.2 & & & & \\
$\quad$ Attentional costs: Sad & -.113 & .226 & -.189 & -0.5 & & & & \\
$\quad$ Condition $\times$ costs: Angry & .366 & .178 & .760 & $2.1^{*}$ & & & & \\
$\quad$ Condition $\times$ costs: Sad & -.095 & .146 & -.250 & -0.65 & & & .17 & 2.3 \\
$\quad$ Total step & & & & & .47 & .22 & .17 \\
\hline
\end{tabular}

Note-Bold factors are those that are entered during that step. $\mathrm{B}$, unstandardized regression coefficient; $\beta$, standardized regression coefficient; $S E$, standard error; Adj. $R^{2}$, adjusted $R^{2} ; F_{\text {inc }}, F$ statistic increment. $\quad{ }^{*} p<$ $.05{ }^{* *} p=.01$. 


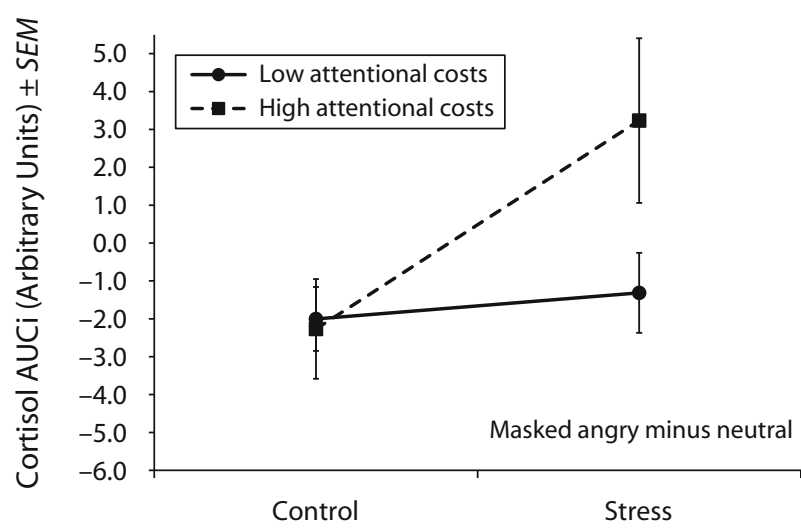

Figure 2. Mean area under the curve with respect to increase (AUCi) by condition (Trier Social Stress Test and control) in participants above (high attentional costs, $n=40$ ) and below (low attentional costs, $n=39$ ) the 50th percentile of the distribution of attentional cost scores, computed by subtracting the response to trials with masked neutral facial expressions from those with masked angry facial expressions. Among participants in the stress condition, those with high attentional costs for masked angry faces exhibited a greater cortisol response than did participants with low attentional costs. No group differences in cortisol change were observed among those in the control condition.

associated with increased cortisol change in response to the TSST relative to the control condition $(t=3.44, p<$ $.005)$. In contrast, the slope for slow attentional shifting toward masked angry faces ( $1 S D$ below the mean) across conditions did not differ from 0 . In sum, the relationship between high attentional costs for masked angry faces and elevated cortisol reactivity during the TSST was largely dependent on attentional engagement as assessed during valid trials. The relationship between low attentional costs for masked sad faces and elevated cortisol reactivity during the experimental procedures was consistent across both valid and invalid trials.

\section{Validity of the Masking Procedure}

A test of awareness was conducted to determine whether participants were consciously aware of the content of masked pictures. The mean (median) percentage of correct identifications of the gender of masked pictures was $55 \%$ $(54 \%)$, indicating that most participants were reporting close to chance level. Among individual participants, the percentage of positive identifications ranged from $30 \%$ to $87 \%$. To determine whether there was a small subgroup of participants exhibiting evidence of awareness of the content of pictures, we examined skewness in the distribution of scores. Positive skewness could indicate that some participants were able to consciously recognize masked pictures. There was slight positive skewness ( \pm standard error of skewness; $0.334 \pm 0.289$ ) on the distribution of correct recognition scores. The exclusion of the 3 participants with the highest picture identifications (all above $80 \%$ correct identifications) removed the positive skewness $(-0.004 \pm 0.291)$. We repeated the regressions described previously in 76 participants, excluding the 3 participants with high recognition scores. There were no differences between these analyses and the previous ones (data not shown). Similar analyses omitting persons who were above $75 \%$ correct in their identification of masked pictures yielded the same results as were observed with the full sample ( $n=74$; data not shown). To maximize power, we opted to present the statistical analyses using the full sample. Thus, these data suggest that most participants could not consciously recognize the content of the masked pictures. A small number of participants may have been able to do so, but this did not alter the relationships presented in the previous sections. For this reason, we refer to the findings as being indicative of "limited conscious awareness," rather than as being "subliminal," "preconscious," or "with no conscious awareness."

\section{DISCUSSION}

The present study sought to replicate and extend a previous study in which we reported that disengagement from masked threatening pictures predicted subsequent cortisol levels collected during a laboratory stress induction and a control session (Ellenbogen, Schwartzman, et al., 2006). A limitation of the previous study was the absence of a stress-induced increase in cortisol in the full sample, despite a robust mood-lowering response. Thus, the present study adopted the TSST - a validated psychosocial stress induction (Dickerson \& Kemeny, 2004). As was expected, the TSST elicited a significant increase in salivary cortisol levels relative to a neutral control condition.

In the present study, two hypotheses were examined. Consistent with our prediction, the interaction between experimental condition and attentional costs on trials with masked angry faces predicted the magnitude of cortisol change during the experiment. The interaction indicated that participants who were exposed to the TSST and who

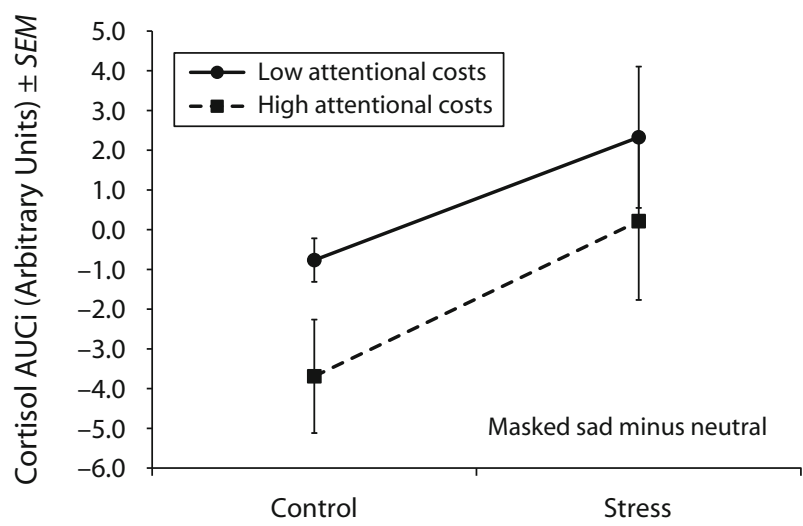

Figure 3. Mean area under the curve with respect to increase (AUCi) by condition (Trier Social Stress Test and control) in participants above (high attentional costs, $n=40$ ) and below (low attentional costs, $n=39$ ) the 50th percentile of the distribution of attentional cost scores for masked sad faces, computed by subtracting the response to trials with masked neutral facial expressions from those with masked sad facial expressions. Attentional costs for masked sad faces were negatively associated with the magnitude of cortisol change. This effect was independent of condition. 
exhibited high attentional costs (i.e., poor attentional flexibility) to masked pictures of angry faces exhibited a robust increase in cortisol levels. In contrast, university students who underwent the stress induction but who displayed low attentional costs (i.e., high attentional flexibility, or avoidance) to masked pictures of angry faces exhibited no increase in cortisol levels. In other words, the automatic processing of pictures depicting threat, occurring with limited conscious awareness, was associated with the degree of HPA reactivity to stress. The third hypothesis predicted that the aforementioned relationship would be driven by abnormalities in the process of disengaging attention from an emotional face, rather than by engaging attention at the cued location. This prediction was not confirmed: A robust interaction between experimental condition and attentional engagement was observed. Disengagement trials alone, in contrast with our previous finding in a clinical population (Ellenbogen, Schwartzman, et al., 2006), were not predictive of cortisol change during stress. Disengagement, therefore, may be less important in healthy university students and more central among clinical populations (Amir et al., 2003; Ellenbogen \& Schwartzman, 2009). In sum, the present study provides a partial replication and extension of our previous study on attentional disengagement and further supports our hypothesis that early emotional information processing may be an important determinant of cortisol reactivity during stress.

The association between emotional information processing and the magnitude of the cortisol response during stress is consistent with a small number of studies examining interactions between attentional factors and HPA functioning (Dandeneau et al., 2007; Roelofs et al., 2007; van Honk et al., 2000). Appelhans and Luecken (2006), for example, observed a positive linear relationship between attentional bias for social threat words and posttask cortisol levels, but only among university students with high trait anxiety. Interestingly, participants with low trait anxiety exhibited the opposite relationship. This study, however, should be interpreted cautiously, since the significant finding is based on a single posttask sample, and the results relate to the processing of words depicting threat. Remarkably, a 5-day attentional training task meant to reduce attentional bias was related to lower cortisol levels during the day in participants who were working in a high-stress environment (Dandeneau et al., 2007). With the exception of one study (Roelofs et al., 2007), none of the past research examines the relationship between emotional information processing during an actual stress induction.

One question that arises from the present study is why cortisol levels were influenced by masked pictures but not by supraliminal pictures, in contrast with the results of the study by van Honk et al. (2000). There are numerous methodological differences between the studies, including the design of the experiment and the information processing task (emotional Stroop vs. spatial cuing). In the present study, it is possible that the presentation of pictures with full conscious awareness elicited other effortful cognitive processes, such as reappraisal or suppression (Gross, 2002), that obscured the relationship between attention and cortisol levels. Cortical circuits, particularly those originating from the prefrontal cortex, are known to regulate the activation of the HPA axis (Diorio, Viau, \& Meaney, 1993; Herman et al., 2003; Kern et al., 2008). It may be that these types of regulatory circuits are activated at later stages of emotional information processing and are therefore bypassed when using masked stimuli.

Because these data are correlational in nature, there are different interpretations of the findings. The relationship between the automatic processing of pictures depicting threat and cortisol levels during acute stress may indicate, as was hypothesized in our model, that attentional shifting regulates, in part, the HPA response to psychosocial stress. Alternatively, these data could be interpreted as indicating that elevated cortisol levels elicited a bias in emotional information processing at an early stage of processing. The latter interpretation is unlikely since the results of the present study were not replicated in a placebo-controlled study of spatial cuing following the administration of 10 and $40 \mathrm{mg}$ of exogenous cortisol (Ellenbogen, Washburn, \& Taylor, 2009). It is important to note, however, that the exogenous administration of cortisol is not equivalent to a laboratory stress induction. Moreover, it is possible that both attentional shifting and cortisol levels are being affected through a third factor, such as stress-related increases in dopamine (Pruessner, Champagne, Meaney, \& Dagher, 2004) or personality traits (Wallace \& Newman, 1998; Wirtz et al., 2007). Future research using experimental manipulations of attention and longitudinal designs are needed to better understand the mechanisms and temporal relationships underlying the present link between automatic information processing and HPA reactivity.

Although the mechanism underlying the relationship between early emotional information processing of threat and stress-induced cortisol reactivity is not known, this relationship is consistent with our previous study (Ellenbogen, Schwartzman, et al., 2006) and with theoretical views of fear processing. Evolutionarily significant information can activate physiological indices of the fear response at an early stage of information processing, prior to conscious awareness (Öhman et al., 2007), through the activation of a subcortical neural circuit between the thalamus and lateral nucleus of the amygdala (LeDoux, 2002; Maratos, Mogg, Bradley, Rippon, \& Senior, 2009; Morris, Öhman, \& Dolan, 1998; Whalen et al., 2004). The coarse stimulus analysis occurring in this circuit (i.e., Whalen et al., 2004) may underlie a biologically prepared response to mobilize the body for action (i.e., increasing blood glucose levels) in the face of threat. The existence of a subcortical circuit devoted to the detection of dangerous and potentially threatening stimuli confers an evolutionary advantage in that defensive responses can be initiated quickly, prior to time-consuming cortical analysis. Because the HPA system is activated, in part, by direct afferents from the central nucleus of the amygdala, it is theoretically possible that the HPA response to threat can be initiated at an early stage of information processing, prior to conscious awareness. Thus, the activation of the HPA axis may be dependent, at least initially, on automatic and preconscious threat evaluation mechanisms residing in subcortical brain areas. 
An unexpected finding in the present study was the robust relationship between attentional engagement and the cortisol response to stress, which is inconsistent with our previous work in this area and its emphasis on disengagement (Ellenbogen, Schwartzman, et al., 2002, 2006). There were a number of methodological differences between the present study and the past ones, including the type of cuing stimuli (facial expressions vs. pictures depicting emotion and emotional words), stress induction (the TSST vs. a repeated loss stressor), and participant population (clinical participants in the community vs. university students). One can only speculate which factor, if any, may have caused the shift from attentional biases in disengagement to engagement. In other studies of spatial cuing, biased attentional engagement on trials using threatening pictures has been observed in clinical depression (Leyman et al., 2007) and in participants with high trait anxiety (Koster et al., 2006). A second unexpected finding was that low attentional costs for masked pictures depicting sadness were associated with greater cortisol reactivity during the experimental procedures. Low attentional costs may represent increased attentional avoidance, in that slow engagement and/or fast disengagement lead to lesser attentional costs. This relationship occurred irrespective of the experimental condition and therefore probably represents a stable, trait-like relationship between the avoidance of sad faces and elevated daytime cortisol levels. Although the meaning of this finding is not known, another study has reported a similar relationship between attentional avoidance and increased cortisol, although only among persons with low trait anxiety levels (Roelofs et al., 2007). Interestingly, we have observed a robust relationship between attentional avoidance and high daytime cortisol levels in the natural environment among a community sample of clinically depressed participants (Ellenbogen \& Ostiguy, 2009). Clearly, there is a need to further examine the correlates and prospective impact of attentional avoidance among different populations.

A few study limitations warrant consideration. First, as was described previously, the present study did not specify the temporal relationship between attentional shifting and stress-related cortisol change, nor can it be ruled out that an unrelated third variable was causing changes to both. Clearly, experimental studies aimed at understanding causal relationships are needed. In particular, attentional retraining studies, which effectively reduce subjective anxiety during acute stress (Amir, Weber, Beard, Taylor, \& Bomyea, 2008), would provide insight into these questions. Second, the masking procedure may have been ineffective in some participants. The recognition data from the picture awareness test were slightly skewed in the positive direction (i.e., the number of participants with high correct recognition scores was slightly higher than the number of participants with low correct recognition scores). Fortunately, this was not a pervasive problem, and reanalyses of the corrected nonskewed data, by removing participants with the highest correct recognition rates, yielded the same results as in the full sample. Still, the present results should be interpreted cautiously with respect to participants' awareness of the content of the masked pictures. Third, although the present study utilized different stimulus exposure durations, the results are dependent on a single RT task, which has been subject to some recent criticism (Mogg et al., 2008). Mogg et al. argued that engagement and disengagement indices may be subject to a confounding influence of general response slowing during trials with affective stimuli. However, the use of attentional costs corrects for this possible methodological limitation. A related issue is whether the present findings are generalizable to other versions of the modified spatial cuing task. For example, the present study utilized a 4:1 ratio of valid to invalid trials, which elicits endogenous (or strategic) shifts of attention. Other studies, in contrast, have used a 1:1 ratio, which assesses exogenous shifts of attention (Koster, Verschuere, Burssens, Custers, \& Crombez, 2007; Leyman et al., 2007). Therefore, future research should focus on different attentional and psychophysiological measures. Finally, the sample size was modest, given the number of predictor variables in some of the regression analyses. Moreover, the study was conducted in a university population, which may not necessarily generalize to the larger community.

In conclusion, attentional shifting toward and away from masked angry faces was a significant predictor of HPA reactivity during acute stress. Although the direction of effect and causal mechanisms are not known, emotional information processing at an early level of processing, with limited conscious awareness, may represent an important step in regulating the magnitude of the HPA response during stress. The importance of understanding these relationships is particularly evident with respect to disorders such as major depression, in which there is evidence of profound HPA dysregulation (Holsboer, 1995) and cognitive biases toward self-relevant and interpersonal stimuli (Ellenbogen \& Schwartzman, 2009; Gotlib et al., 2004), some of which likely precede the development of the disorder (Ellenbogen, Hodgins, Walker, Adam, \& Couture, 2006; Ellenbogen, Santo, Linnen, Walker, \& Hodgins, 2010; Joormann, Talbot, \& Gotlib, 2007). It is likely that deficits in emotional information processing (Leppänen, 2006), amygdala functioning (Ramel et al., 2007), and left prefrontal-amygdala regulatory circuits (Johnstone, van Reekum, Urry, Kalin, \& Davidson, 2007) are linked to HPA dysfunction in depression; a better understanding of these relationships will provide new insight into the pathophysiology of this disorder and possibly new modes of therapeutic intervention.

\section{AUTHOR NOTE}

The present work was supported by research funds from the Canada Research Chair program (through the Social Sciences and Humanities Research Council of Canada), awarded to M.A.E. We thank Yara Kadulina and George Michael Everett for their invaluable assistance on the project. Address correspondence to M. A. Ellenbogen, Centre for Research in Human Development, Concordia University, 7141 Sherbrooke St. West, Montreal, QC, H4B 1R6 Canada (e-mail: mark .ellenbogen@concordia.ca). 


\section{REFERENCES}

Aiken, L. S., \& West, S. G. (1991). Multiple regression: Testing and interpreting interactions. Newbury Park, CA: Sage.

Amir, N., Elias, J., Klumpr, H., \& Przeworski, A. (2003). Attentional bias to threat in social phobia: Facilitated processing of threat or difficulty disengaging attention from threat? Behaviour Research \& Therapy, 41, 1325-1335.

Amir, N., Weber, G., Beard, C., Taylor, C. T., \& Bomyea, J. (2008). The effect of a single-session attention modification program on response to a public-speaking challenge in socially anxious individuals. Journal of Abnormal Psychology, 117, 860-868.

Appelhans, B. M., \& Luecken, L. J. (2006). Attentional processes, anxiety, and the regulation of cortisol reactivity. Anxiety, Stress \& Coping, 19, 81-92.

BARGH, J. A. (1989). Conditional automaticity: Varieties of automatic influence in social perception and cognition. In J. S. Uleman \& J. A. Bargh (Eds.), Unintended thought (pp. 3-51). New York: Guilford.

BARGH, J. A. (2006). Agenda 2006: What have we been priming all these years? On the development, mechanisms, and ecology of nonconscious social behavior. European Journal of Social Psychology, 36, 147-168.

Beck, A. T., Steer, R. A., \& Brown, G. K. (1996). Manual for the Beck Depression Inventory-II. San Antonio, TX: Psychological Corporation.

Beevers, C. G., \& Carver, C. S. (2003). Attentional bias and mood persistence as prospective predictors of dysphoria. Cognitive Therapy \& Research, 27, 619-637.

BREIER, A. (1989). Experimental approaches to human stress research: Assessment of neurobiological mechanisms of stress in volunteers and psychiatric patients. Biological Psychiatry, 26, 438-462.

Buchanan, T. W., \& Lovallo, W. R. (2001). Enhanced memory for emotional material following stress-level cortisol treatment in humans. Psychoneuroendocrinology, 26, 307-317.

Calvo, M. G., \& Avero, P. (2005). Time course of attentional bias to emotional scenes in anxiety: Gaze direction and duration. Cognition \& Emotion, 19, 433-451.

CAMPBell-Sills, L., \& BARLOW, D. H. (2007). Incorporating emotion regulation into conceptualizations and treatments of anxiety and mood disorders. In J. Gross (Ed.), Handbook of emotion regulation (pp. 542559). New York: Guilford.

Compton, R. J. (2000). Ability to disengage attention predicts negative affect. Cognition \& Emotion, 14, 401-415.

Dandeneau, S. D., Baldwin, M. W., Baccus, J. R., SakellaroPOUlo, M., \& Pruessner, J. C. (2007). Cutting stress off at the pass: Reducing vigilance and responsiveness to social threat by manipulating attention. Journal of Personality \& Social Psychology, 93, 651-666.

Dickerson, S. S., \& Kemeny, M. E. (2004). Acute stressors and cortisol responses: A theoretical integration and synthesis of laboratory research. Psychological Bulletin, 130, 355-391.

Diorio, D., Viau, V., \& Meaney, M. J. (1993). The role of the medial prefrontal cortex (cingulate gyrus) in the regulation of hypothalamicpituitary-adrenal responses to stress. Journal of Neuroscience, 13, 3839-3847.

Ekman, P., \& Friesen, W. V. (1976). Pictures of facial affect. San Francisco: University of California, Human Interactions Laboratory.

Ellenbogen, M. A., Hodgins, S., Walker, C.-D., Adam, S., \& CouTURE, S. (2006). Daytime cortisol and stress reactivity in the offspring of parents with bipolar disorder. Psychoneuroendocrinology, 31, 1164-1180.

Ellenbogen, M. A., \& Ostiguy, C. (2009). [Attentional disengagement, stress, and salivary cortiol levels in the natural environment among clinically depressed participants]. Unpublished raw data.

Ellenbogen, M. A., Santo, J. B., Linnen, A.-M., Walker, C.-D., \& Hodgins, S. (2010). High cortisol levels in the offspring of parents with bipolar disorder during two weeks of daily sampling. Bipolar Disorders, 12, 77-86.

Ellenbogen, M. A., \& Schwartzman, A. E. (2009). Selective attention and avoidance on a pictorial cueing task during stress in clinically anxious and depressed participants. Behaviour Research \& Therapy, 47, 128-138.

Ellenbogen, M. A., Schwartzman, A. E., Stewart, J., \& Walker, C.-D. (2002). Stress and selective attention: The interplay of mood, cortisol levels, and emotional information processing. Psychophysiology, 39, 723-732.

Ellenbogen, M. A., Schwartzman, A. E., Stewart, J., \& Walker, C.-D. (2006). Automatic and effortful emotional information processing regulates different aspects of the stress response. Psychoneuroendocrinology, 31, 373-387.

Ellenbogen, M. A., Washburn, D., \& Taylor, V. (2009). The effects of exogenous glucocorticoids on attentional disengagement from emotional pictures. Unpublished manuscript.

Fales, C. L., Barch, D. M., Rundle, M. M., Mintun, M. A., Snyder, A. Z., Cohen, J. D., ET AL. (2008). Altered emotional interference processing in affective and cognitive-control brain circuitry in major depression. Biological Psychiatry, 63, 377-384.

Fehm-Wolfsdorf, G., Soherr, U., Arndt, R., Kern, W., Fehm, H. L., \& Nagel, D. (1993). Auditory reflex thresholds elevated by stressinduced cortisol secretion. Psychoneuroendocrinology, 18, 579-589.

Fox, E., Russo, R., Bowles, R., \& Dutton, K. (2001). Do threatening stimuli draw or hold visual attention in subclinical anxiety? Journal of Experimental Psychology: General, 130, 681-700.

GaAb, J., Rohleder, N., Nater, U. M., \& Ehlert, U. (2005). Psychological determinants of the cortisol stress response: The role of anticipatory cognitive appraisal. Psychoneuroendocrinology, 30, 599-610.

Gotlib, I. H., Krasnoperova, E., Yue, D. N., \& Joormann, J. (2004). Attentional biases for negative interpersonal stimuli in clinical depression. Journal of Abnormal Psychology, 113, 127-135.

Gross, J. J. (1998). The emerging field of emotion regulation: An integrative review. Review of General Psychology, 2, 271-299.

Gross, J. J. (2002). Emotion regulation: Affective, cognitive, and social consequences. Psychophysiology, 39, 281-291.

Herman, J. P., Figueiredo, H., Mueller, N. K., Ulrich-Lai, Y., OsTrander, M. M., Choi, D. C., \& Cullinan, W. E. (2003). Central mechanisms of stress integration: Hierarchical circuitry controlling hypothalamo-pituitary-adrenocortical responsiveness. Frontiers in Neuroendocrinology, 24, 151-180.

Holsboer, F. (1995). Neuroendocrinology of mood disorders. In F. E. Bloom \& D. J. Kupfer (Eds.), Psychopharmacology: The fourth generation of progress (pp. 957-969). New York: Raven.

Ingram, R. E., Miranda, J., \& Segal, Z. (2006). Cognitive vulnerability to emotional disorders. In L. B. Alloy \& J. H. Riskind (Eds.), Cognitive vulnerability to depression. Mahwah, NJ: Erlbaum.

Johnstone, T., van Reekum, C. M., Urry, H. L., Kalin, N. H., \& DAVIDSON, R. J. (2007). Failure to regulate: Counterproductive recruitment of top-down prefrontal-subcortical circuitry in major depression. Journal of Neuroscience, 27, 8877-8884.

Joormann, J., Siemer, M., \& Gotlib, I. H. (2007). Mood regulation in depression: Differential effects of distraction and recall of happy memories on sad mood. Journal of Abnormal Psychology, 116, 484490.

Joormann, J., Talbot, L., \& Gotlib, I. H. (2007). Biased processing of emotional information in girls at risk for depression. Journal of Abnormal Psychology, 116, 135-143.

Kern, S., Oakes, T. R., Stone, C. K., McAuliff, E. M., KirschBAUM, C., \& Davidson, R. J. (2008). Glucose metabolic changes in the prefrontal cortex are associated with HPA axis response to a psychosocial stressor. Psychoneuroendocrinology, 33, 517-529.

Kirschbaum, C., Pirke, K.-M., \& Hellhammer, D. H. (1993). The "Trier Social Stress Test": A tool for investigating psychobiological stress responses in a laboratory setting. Neuropsychobiology, 28, 7681.

Koster, E. H. W., Crombez, G., Verschuere, B., Van Damme, S., \& Wiersema, J. R. (2006). Components of attentional bias to threat in high trait anxiety: Facilitated engagement, impaired disengagement, and attentional avoidance. Behaviour Research \& Therapy, 44, 17571771.

Koster, E. H. W., De Raedt, R., Goeleven, E., Franck, E., \& CromBEZ, G. (2005). Mood-congruent attentional bias in dysphoria: Maintained attention to and impaired disengagement from negative information. Emotion, 5, 446-455.

Koster, E. H. W., Verschuere, B., Burssens, B., Custers, R., \& Crombez, G. (2007). Attention for emotional faces under restricted awareness revisited: Do emotional faces automatically attract attention? Emotion, 7, 285-295.

KRING, A. M., \& Werner, K. H. (2004). Emotion regulation and psy- 
chopathology. In P. Philippot \& R. S. Feldman (Eds.), The regulation of emotion (pp. 359-385). Mahwah, NJ: Erlbaum.

LaZarus, R. S., \& FolKman, S. (1984). Stress, appraisal, and coping. New York: Springer.

LeDoux, J. E. (2002). Fear and the brain: Where have we been, and where are we going? In J. T. Cacioppo, G. G. Berntson, R. Adolphs, C. S. Carter, R. J. Davidson, M. K. McClintock, et al. (Eds.), Foundations in social neuroscience (pp. 411-423). Cambridge, MA: MIT Press.

LEPPÄNEN, J. (2006). Emotional information processing in mood disorders: A review of behavioral and neuroimaging findings. Current Opinion in Psychiatry, 19, 34-39.

Leyman, L., De Raedt, R., Schacht, R., \& Koster, E. H. W. (2007). Attentional biases for angry faces in unipolar depression. Psychological Medicine, 37, 393-402.

LogAN, G. D. (1992). Attention and preattention in theories of automaticity. American Journal of Psychology, 105, 317-339.

Luck, S. J., Hillyard, S. A., Mouloua, M., WoldorfF, M. G., Clark, V. P., \& HawkINS, H. L. (1994). Effects of spatial cuing on luminance detectability: Psychophysical and electrophysiological evidence for early selection. Journal of Experimental Psychology: Human Perception \& Performance, 20, 887-904.

LupIen, S. J., \& McEwEn, B. S. (1997). The acute effects of corticosteroids on cognition: Integration of animal and human model studies. Brain Research Reviews, 24, 1-27.

Maratos, F. A., Mogg, K., Bradley, B. P., Rippon, G., \& Senior, C. (2009). Coarse threat images reveal theta oscillations in the amygdala: A magnetoencephalography study. Cognitive, Affective, \& Behavioral Neuroscience, 9, 133-143.

Mathews, A., \& MACLEOD, C. (2002). Induced processing biases have causal effects on anxiety. Cognition \& Emotion, 16, 331-354.

McEwen, B. S. (2004). Protection and damage from acute and chronic stress: Allostasis and allostatic overload and relevance to the pathophysiology of psychiatric disorders. Annals of the New York Academy of Sciences, 1032, 1-7.

McLaughlin, K. A., Mennin, D. S., \& Farach, F. J. (2007). The contributory role of worry in emotion generation and dysregulation in generalized anxiety disorder. Behaviour Research \& Therapy, 45, 1735-1752.

Meyer, T. J., Miller, M. L., Metzger, R. L., \& Borkovec, T. D. (1990). Development and validation of the Penn State Worry Questionnaire. Behaviour Research \& Therapy, 28, 487-495.

MogG, K., \& Bradley, B. P. (1998). A cognitive-motivational analysis of anxiety. Behaviour Research \& Therapy, 36, 809-848.

Mogg, K., \& Bradley, B. P. (2005). Attentional bias in generalized anxiety disorder versus depressive disorder. Cognitive Therapy \& Research, 29, 29-45.

Mogg, K., Holmes, A., Garner, M., \& Bradley, B. P. (2008). Effects of threat cues on attentional shifting, disengagement and response slowing in anxious individuals. Behaviour Research \& Therapy, 46, 656-667.

Mölle, M., Albrecht, C., Marshall, L., Fehm, H. L., \& Born, J. (1997). Adrenocorticotropin widens the focus of attention in humans. A nonlinear electroencephalographic analysis. Psychosomatic Medicine, 59, 497-502.

Morris, J. S., Öhman, A., \& Dolan, R. J. (1998). Conscious and unconscious emotional learning in the human amygdala. Nature, 393, 467-470.

Öhman, A., Carlsson, K., Lundevist, D., \& IngVar, M. (2007). On the unconscious subcortical origin of human fear. Physiology \& Behavior, 92, 180-185.

Öhman, A., \& Soares, J. J. F. (1994). "Unconscious anxiety": Phobic responses to masked stimuli. Journal of Abnormal Psychology, 103, 231-240.

Posner, M. I. (1978). Chronometric explorations of mind. Hillsdale, NJ: Erlbaum.

Posner, M. I., Nissen, M. J., \& Ogden, W. C. (1978). Attended and unattended processing modes: The role of set for spatial location. In H. L. Pick Jr. \& E. Saltzman (Eds.), Modes of perceiving and processing information (pp. 137-157). Hillsdale, NJ: Erlbaum.

Posner, M. I., \& Rothbart, M. K. (1998). Attention, self-regulation and consciousness. Philosophical Transactions of the Royal Society B, 353, 1915-1927.

Posner, M. I., Snyder, C. R. R., \& Davidson, B. J. (1980). Attention and the detection of signals. Journal of Experimental Psychology: General, 109, 160-174.

Pruessner, J. C., Champagne, F., Meaney, M. J., \& Dagher, A. (2004). Dopamine release in response to a psychological stress in humans and its relationship to early life maternal care: A positron emission tomography study using $\left[{ }^{11} \mathrm{c}\right]$ raclopride. Journal of Neuroscience, 24, 2825-2831.

Pruessner, J. C., Kirschbaum, C., Meinlschmid, G., \& HellhamMER, D. H. (2003). Two formulas for computation of the area under the curve represent measures of total hormone concentration versus time-dependent change. Psychoneuroendocrinology, 28, 916-931.

Putman, P., Hermans, E. J., Koppeschaar, H. P., van Schijndel, A., \& VAN HONK, J. (2007). A single administration of cortisol acutely reduces the preconscious bias attention for fear in anxious young men. Psychoneuroendocrinology, 32, 793-802.

Putman, P., van Honk, J., Kessels, R. P. C., Mulder, M., \& KoppeSCHAAR, H. P. F. (2004). Salivary cortisol and short and long-term memory for emotional faces in healthy young women. Psychoneuroendocrinology, 29, 953-960.

Ramel, W., Goldin, P. R., Eyler, L. T., Brown, G. G., Gotlib, I. H., \& MCQUAID, J. R. (2007). Amygdala reactivity and mood-congruent memory in individuals at risk for depressive relapse. Biological Psychiatry, 61, 231-239.

Roelofs, K., Bakvis, P., Hermans, E. J., van Pelt, J., \& van Honk, J. (2007). The effects of social stress and cortisol responses on the preconscious selective attention to social threat. Biological Psychology, 75, 1-7.

Salemink, E., van den Hout, M. A., \& Kindt, M. (2007). Selective attention and threat: Quick orienting versus slow disengagement and two versions of the dot probe task. Behaviour Research \& Therapy, 45, 607-615.

Schwartz, E. P., Granger, D. A., Susman, E. J., Gunnar, M. R., \& LAIRD, B. (1998). Assessing salivary cortisol in studies of child development. Child Development, 69, 1503-1513.

Shiffrin, R. M., \& SchneIder, W. (1977). Controlled and automatic human information processing: II. Perceptual learning, automatic attending and a general theory. Psychological Review, 84, 127-190.

Skosnik, P. D., Chatterton, R. T., JR., Swisher, T., \& PArk, S. (2000). Modulation of attentional inhibition by norepinephrine and cortisol after psychological stress. International Journal of Psychophysiology, 36, 59-68.

Sposari, J., \& RAPEe, R. (2007). Attentional bias toward facial stimuli under conditions of social threat in socially phobic and nonclinical participants. Cognitive Therapy \& Research, 31, 23-37.

Stormark, K. M., Nordby, H., \& Hugdahl, K. (1995). Attentional shifts to emotionally charged cues: Behavioural and ERP data. Cognition \& Emotion, 9, 507-523.

VAN ECK, M. M., \& NICOLSON, N. A. (1994). Perceived stress and salivary cortisol in daily life. Annals of Behavioral Medicine, 16, 221-227.

van Honk, J., Tuiten, A., van den Hout, M., Koppeschaar, H., Thijssen, J., de HaAn, E., \& Verbaten, R. (2000). Conscious and preconscious selective attention to social threat: Different neuroendocrine response patterns. Psychoneuroendocrinology, 25, 577591.

Wallace, J. F., \& Newman, J. P. (1998). Neuroticism and the facilitation of the automatic orienting of attention. Personality \& Individual Differences, 24, 253-266.

Whalen, P. J., Kagan, J., Cook, R. G., Davis, F. C., Kim, H., Polis, S., ET AL. (2004). Human amygdala responsivity to masked fearful eye whites. Science, 306, 2061.

Wirtz, P. H., Elsenbruch, S., Emini, L., Rüdisüli, K., GroessBAUer, S., \& Ehlert, U. (2007). Perfectionism and the cortisol response to psychosocial stress in men. Psychosomatic Medicine, 69, 249-255.

Young, A. H. (2004). Cortisol in mood disorders. Stress, 7, 205-208.

(Manuscript received May 22, 2009;

revision accepted for publication September 24, 2009.) 